\title{
Radiological and surgical aspects of round window visibility during cochlear implantation: a retrospective analysis
}

\author{
Saad Jwair ${ }^{1,2}$ (1) · Job J. M. van Eijden ${ }^{1}$ - Esther E. Blijleven ${ }^{1}$ - Jan Willem Dankbaar ${ }^{3} \cdot$ Hans G. X. M. Thomeer ${ }^{1,2}$
}

Received: 3 November 2020 / Accepted: 5 January 2021 / Published online: 20 January 2021

(c) The Author(s) 2021

\begin{abstract}
Purpose The round window approach has become the most preferred option for cochlear implant (CI) insertion, however, sometimes it may not be possible due to the (in)visibility of the round window membrane (RWM). We addressed the prevalence, consequences and indicators of difficult detection of the RWM in cochlear implant surgery.

Methods This study retrospectively analysed the operative reports and preoperative high resolution axial-computed tomography (CT) scans of a consecutive cohort of patients who underwent a CI insertion. The main outcomes were surgical outcomes of the RW approach, and assessment of radiologicalmarkers.

Results The operative reports showed that RWM insertion was feasible in 151 out of 153 patients. In $18 \%$ of the patients the RWM was difficult to visualize. All these patients had at least one intraoperative event. The chorda tympani nerve (CTN) or posterior canal wall was affected in $8 \%$ of the 153 patients and the fallopian canal in $6 \%$. These patients had a facial-chorda tympani nerve distance on the CT scan that was considerably smaller than normal patients (1.5 $\mathrm{mm}$ vs $2.3 \mathrm{~mm}$ ). In addition, a prediction line towards the anterolateral side of the RWM was found to be more prevalent in these patients' CT scans (sensitivity $81 \%$, specificity $63 \%$ ).

Conclusion The RW approach is feasible in almost all patients undergoing CI surgery. Difficult visualisation of the RWM seems to lead to at least one intraoperative event. Radiological measures showed that these patients had a smaller facial recess and a more anteriorly placed facial nerve, which can be used to better plan a safe insertion approach.
\end{abstract}

Keywords Auditory prostheses $\cdot$ Cochlear implant $\cdot$ Round window $\cdot$ Facial nerve $\cdot$ Chorda tympani nerve

\section{Introduction}

Cochlear implants (CIs) provide a solution for patients of all ages with severely impaired hearing. The classical surgical method of implantation is performed by way of a retro-auricular approach with a mastoidectomy-facial recess technique, followed by a CI insertion via either the round window membrane (RWM) or an anteroinferiorly (relative to

Saad Jwair

s.jwair@umcutrecht.nl

1 Department of Otorhinolaryngology and Head \& Neck Surgery, University Medical Center Utrecht, Utrecht University, Heidelberglaan 100, P.O. Box 85500, 3508 GA Utrecht, The Netherlands

2 UMC Utrecht Brain Center, Utrecht University, Utrecht, The Netherlands

3 Department of Radiology, University Medical Center Utrecht, PO Box 85500, 3508 GA Utrecht, The Netherlands the RWM) placed cochleostomy [1,2]. This surgical method is standard care in most CI centres worldwide [3]. The RW approach is nowadays normally preferred over a cochleostomy because it might be less traumatic [1, 4].

Although the RW approach is widely adopted, only few studies reported its feasibility and complications $[3,5]$. The RW approach is not always possible, presumably because of the sometimes difficult visualisation of the RWM $[2,3,6]$. Intraoperatively, trying to improve visibility of the RWM can lead to an increased chance of intentional or unintentional damage to important structures like the chorda tympani nerve $(\mathrm{CTN})$, the fallopian canal, posterior canal wall or tympanic membrane. Although this damage does not necessarily lead to postoperative complications, it is preferred to leave these structures intact [7]. To avoid these situations, it might be beneficial to assess the RWM visibility before surgery.

In current medical practice RWM visibility is not assessed beforehand. A preoperative high resolution computed 
tomography (HRCT) is used to assess medical contraindications for a RW approach (e.g. otosclerosis or cochlear malformations) [8]. In addition, surgeons use this scan to be adequately prepared for surgery, by assessing important surgical landmarks such as the sigmoid sinus, incus and lateral semicircular canal $[8,9]$. Previous studies have shown that these scans can also be used for investigation of the RWM visibility [10-13].

For this study we outlined two goals regarding cochlear implantation surgery: (1) to identify the feasibility of the RW approach in our adult population, and (2) to assess the prevalence, consequences and radiological markers of difficult RWM visibility.

\section{Materials and methods}

\section{Study design}

The operative reports and preoperative HRCT scans of a cohort of adult patients that received a CI at our tertiary referral centre between January 2015 and March 2020 were retrospectively examined. These patients were consecutively operated by one surgeon. The data were collected from the patient files. The eligibility criteria were as follows: (1) age $\geq 18$ years, (2) no inner ear deformities, (3) primary cochlear implantation, (4) no prior mastoid or middle ear surgery on the implanted side, (5) no signs of (chronic) otomastoiditis, (6) patent RWM and scala tympani (ST) of implanted side on preoperative HRCT scan. The first five items were assessed with the operative and medical report data. If discussion on the eligibility criteria was encountered, consensus was obtained between the authors.

\section{Operative report}

The operative report of every patient of the database was evaluated by two investigators ( $\mathrm{SJ}$ and JvE). The following variables were extracted: age, gender, medical diagnosis, side of implantation, type of middle ear and insertion approach, mastoid pneumatisation, view of RWM (easy or difficult), facial recess size (normal or small), other notable issues (e.g. overhanging posterior wall or bulging jugular bulb), and lastly intraoperative events (e.g. lesions of the $\mathrm{CTN}$, posterior wall, facial nerve (FN) and fallopian canal). In addition, the postoperative medical reports of cases with an intraoperative event involving the $\mathrm{FN}$ or CTN were reviewed for related complaints (e.g. tongue sensitization or face paralysis).

\section{High resolution CT scan}

High resolution temporal bone images (axial and coronal plane reconstructions) with a slice thickness of $1.0 \mathrm{~mm}$ were obtained using a Siemens-force CT scanner at $120 \mathrm{kV}$ and $150 \mathrm{mAs}$ or a Philips scanner at $120 \mathrm{kV} 300 \mathrm{mAs}$. Two investigators ( $\mathrm{SJ}$ and $\mathrm{JvE}$ ) analysed and gathered the HRCT scans. These investigators were not involved in any of the surgeries, and were blinded for the operative findings during the analysis of the HRCT scans. Beforehand, the investigators were trained by an ENT surgeon (HT) and neuroradiologist (JWD) in the analysis of the mastoid, with an extra focus on the course of the FN and CTN.

The CTN was identified by three points:

\section{Origin of the FN at mastoid tip}

2. Mastoidal course until tympanic annulus (bony rim of the tympanic membrane)

3. Re-appearing again at the anterior wall of the middle ear cavity and entering the petrotympanic fissure.

The authors drew a line between the FN and the CTN on the axial HRCT scan, see Fig. 1. The measurement of the FN-CTN distance was defined by the shortest distance (inner margin) between two points on the axial HRCT
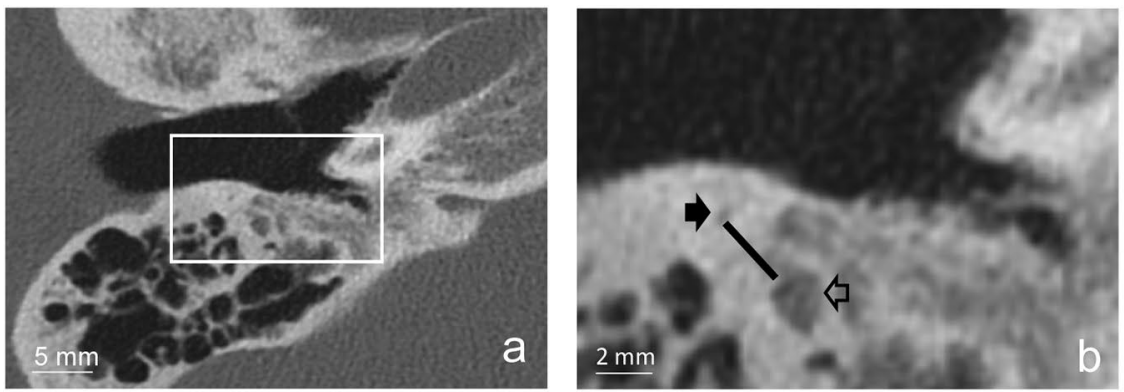

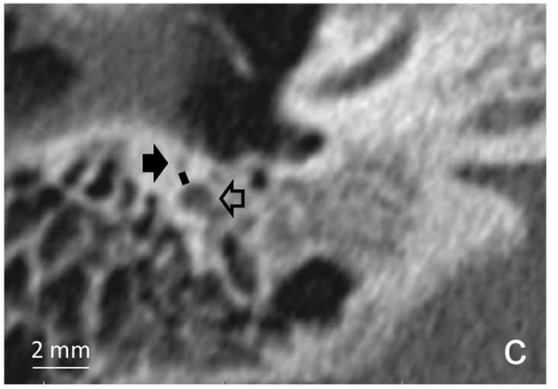

Fig. 1 a Overview of the preoperative axial high resolution CT scan of the right temporal bone. b Magnification $(\times 2.5)$ of the same axial high resolution $\mathrm{CT}$ scan of the right temporal bone. Black arrow depicts the chorda tympani nerve (CTN), and the unfilled arrow the facial nerve (FN). The line between these two nerves is the FN-CTN distance. This case had a FN-CTN distance of $2.9 \mathrm{~mm}$. c Axial high resolution CT scan of the right temporal bone of another patient. This case had a small FN-CTN distance of $0.6 \mathrm{~mm}$ 
reconstructions with the posterior canal wall/mastoid and the middle ear space within the same plane:

1. The CTN, as close as possible to its entry in the middle ear space, but still in the mastoid.

2. FN, at the point of the second genu.

The second last axial HRCT section of the mastoid segment of the CTN, before entering the middle ear space, proved to be the most optimal section to measure the FNCTN distance. This measurement enabled us to confidently state the near maximal distance of the facial recess opening between the FN and CTN.

The authors established a second measurement, partly based on a previous study [12], that indicated the anterior position of the FN relative to the RWM. A prediction line was drawn from the anterior part of the mastoid course of the FN on the axial planes, towards the lower side of the basal turn of the cochlea. Subsequently, the intersection point between the RWM and the prediction line is categorized in being either anterolateral or posteromedial, see Fig. 2. All intersection points below the middle of the RWM were classified as posteromedial, and the intersection points above this middle were classified as anterolateral.

\section{Analysis}

Based on the operative reports we established whether the intended RWM insertion was successful. The operative reports were also used to assess the intraoperative visibility of the RWM. Two groups were identified: cases with normal identification of the RWM and cases with difficult visibility of the RWM. Cases with difficult RW niche visibility were also included in the latter group. After excluding all cases with inadequate scans we compared the radiological measurements between the normal and difficult cases. For the second radiological measurement (i.e. prediction line) 20 cases of the normal group, at random, were selected for the comparison analysis. All radiological analyses were done blinded for the operative report and outcomes.

\section{Results}

The patient cohort, January 2015-May 2020, was screened for the in- and exclusion criteria (see Fig. 3). After applying these criteria, 153 cases were included for the operative report analysis. Regarding the HRCT analyses, we had to exclude 33 from 153 cases, 30 from the normal group and 3 from the difficult group, because in those cases the only available scan was of a low-quality $\mathrm{CT}$ with an inadequate image resolution or with severe motion artefacts. In total, 120 HRCTs were analysed.

\section{Operative report}

In 151 out of 153 patients (99\%) a RWM insertion was realized and successful, the other two patients received a cochleostomy. An example of the intraoperative view is depicted in Fig. 4. That example would classify as a normal case, as the surgeon is able to identify the RWM with intact anatomical borders (i.e. FN, CTN, incus buttress and posterior canal wall). The patient characteristics and intraoperative events are summarized in Table 1.

In total, in 28 patients (18\% from total), the RWM and niche detection was difficult, mostly due to a small facial recess (26/28). In one case, the posterior canal wall was hindering the surgeons view, while another case had a high riding jugular bulb obstructing the RWM access. Interestingly, all these patients had at least one intraoperative
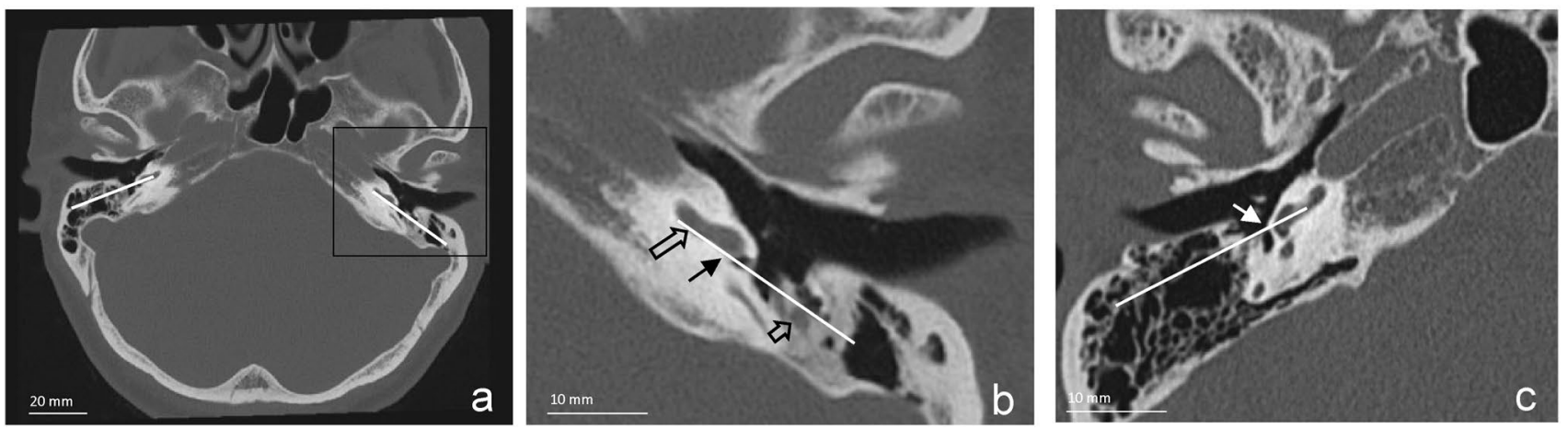

Fig. 2 a Prediction line drawn on the axial high resolution scan of both temporal bones. $\mathbf{b}$ Close up view of the prediction line. The prediction line was drawn on the preoperative axial high resolution CT scans between the anterolateral mastoid facial nerve and the lower side of the basal turn of the cochlea. The intersection point lies on the posteromedial side of the round window membrane in this example. Large unfilled arrow $=$ lower side of basal turn, filled black arrow $=$ posteromedial intersection point, small unfilled arrow $=$ facial nerve. c Example of an intersection point on the anterolateral side of the RWM. White arrow = anterolateral intersection point 
262 consecutive $\mathrm{Cl}$ cases from cohort 2015-2020

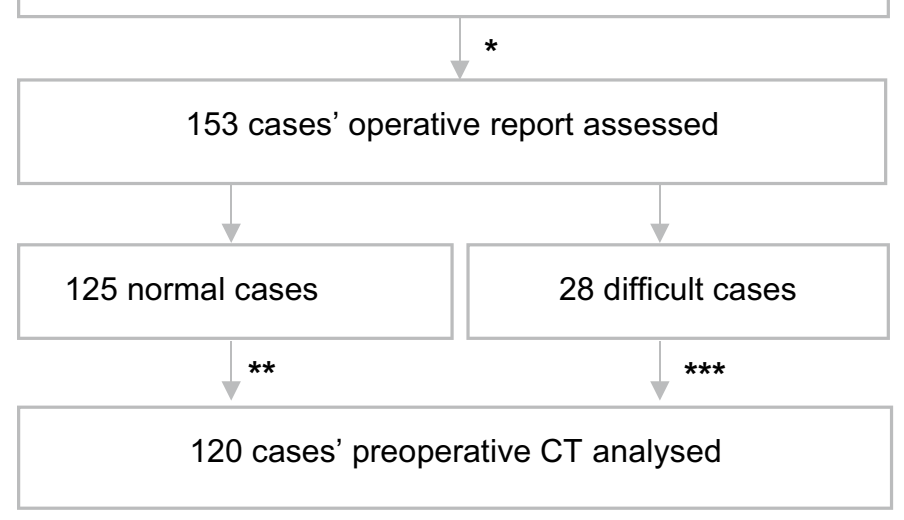

109 cases excluded:

- $\quad$ Age $\leq 18$ years

- Secondary implant surgery

- Prior mastoid surgery on implanted side

- Otomastoiditis

- Inner ear deformities

- $\quad$ Non patent RW membrane/scala tympani

$* *$

30 cases excluded due to inadequate low resolution $\mathrm{CT}$ scans or motion artefacts

***

3 cases excluded due to inadequate low resolution CT scans or motion artefacts

Fig. 3 Flow chart of the in- and excluded cases for both the operative report and preoperative computed tomography scan analyses
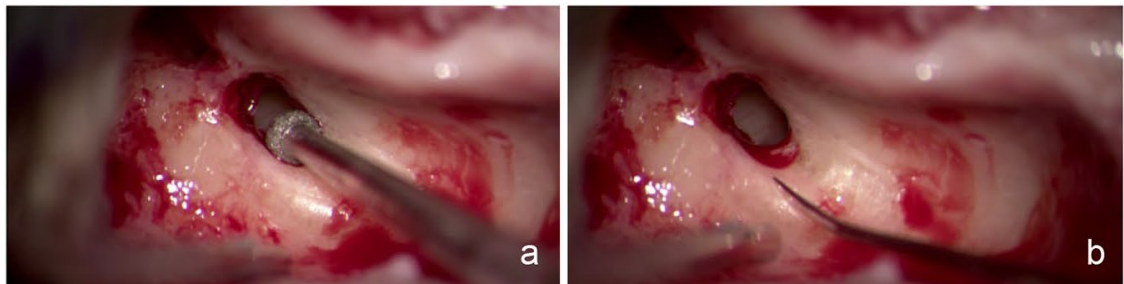

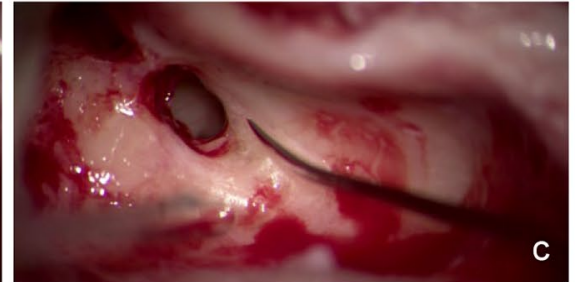

Fig. 4 a Intraoperative view of the facial recess opening (right ear), a $2 \mathrm{~mm}$ burr fits easily in the facial recess opening. b Facial nerve is clearly identifiable, with an intact posterior canal wall. c Chorda

event. The chorda tympani nerve (CTN) was sacrificed in 13 cases (8\%), posterior canal wall lesions in 12 cases (8\%) and fallopian canal uncovering in 10 cases (6\%). The CTN had to be sacrificed to provide adequate visualisation of the RWM and niche. Postoperative medical reports showed no complaints related to the CTN sacrifice (e.g. taste disturbance or tongue sensitization). Furthermore, to improve the visibility through the facial recess opening, a small part of the bony cover of the FN canal had to be removed. No FN weakness direct postoperatively or long term was noted in any case. Finally, no complaints were detected for patients with the partial uncovering of the bony posterior wall of the external auditory canal or tympanic membrane annulus.

The intraoperative findings were evenly spread over time (during the included period of investigation) and side of implantation, indicating no relationship of these findings with either of these factors. tympani nerve is also clearly identifiable, the round window is seen posteroinferiorly in the facial recess opening

\section{High resolution CT scan}

In total, 120 HRCT scans were analysed for the FN-CTN distance, and divided in two groups based on the operative reports: 95 scans of the normal cases, and 25 scans of the difficult cases. For the prediction line, 20 cases of the normal group, at random, were selected for comparative analysis with the difficult cases. A sclerotic mastoid was seen in $10 \%$ of the patients (in concordance with the operative reports), no difference was observed between both groups.

\section{Facial-chorda tympani nerve distance}

The mean FN-CTN distance was $2.2 \mathrm{~mm}$ (SD: 0.5, confidence interval 2.12-2.32) for the normal cases $(n=95)$, in contrast, the mean distance was $1.5 \mathrm{~mm}$ (SD: 0.4 , confidence interval 1.31-1.68) for cases with difficult view of the RWM $(n=23)$, which is a significant difference ( $t$ test, $p<0.001$ ). 
Table 1 Patient characteristics and outcomes, $n=153(\%)$

\begin{tabular}{lc}
\hline Age at implantation, years (SD) & $62(16)$ \\
Gender & $80(52)$ \\
Male & $73(48)$ \\
Female & \\
Ethnicity & $149(97)$ \\
Native Dutch & \\
Diagnosis & $151(99)$ \\
Progressive bilateral SNHL & \\
Side of implantation & $72(47)$ \\
Right & $80(52)$ \\
Left & $1(<1)$ \\
Bilateral & $14(9)$ \\
Mastoid pneumatization & \\
Sclerotic & $153(100)$ \\
Type of middle ear approach & \\
Mastoidectomy-facial recess & $151(99)$ \\
Type of insertion approach & $2(1)$ \\
Direct RWM & $10(6)$ \\
Cochleostomy & $13(8)$ \\
Intraoperative events & \\
Facial nerve exposure & $12(8)$ \\
Chorda tympani nerve lesion & \\
EAM/TM lesion & \\
Other & \\
\hline
\end{tabular}

EAM external auditory meatus, $T M$ tympanic membrane, $R W M$ round window membrane, $S D$ standard deviation

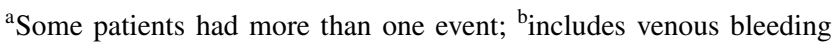
and tegmen tympani lesions
The FN-CTN distance of $\leq 1.5 \mathrm{~mm}$ was applicable for 9 patients (9\%) of the normal group, and for 17 patients (74\%) of the difficult cases group, resulting in a sensitivity of $65 \%$ and a specificity of $93 \%$. Two cases with a difficult view of the RWM were left out of this analysis, because the visibility of the RWM was hindered by other factors than the facial recess opening. The FN-CTN distance was $2.9 \mathrm{~mm}$ for one case with an overhanging posterior canal wall, and the other case had a high riding jugular bulb with a FN-CTN distance of $2.2 \mathrm{~mm}$.

\section{Prediction line}

Axial HRCT reconstructions showed that the anterolateral FN and the basal turn of the cochlea could not be reliable identified in 3 out of 23 cases with a difficult view of the RWM and niche. Those cases were therefore excluded, resulting in 20 included cases with difficult view of the RWM. Analysis showed that in group A (difficult cases) 9 out of 20 had an anterolateral intersection point, and 11 out of 20 had a posteromedial intersection point. For group B (normal cases) 3 out of 20 had an anterolateral intersection point, and 17 out of 20 had a posteromedial intersection point. See Fig. 5 for a summary of these results. The sensitivity was $81 \%$, and specificity $63 \%$, with a posteromedial intersection point being favourable for easy or normal detection of the RWM. No differences were observed between both sides within cases.
Fig. 5 Comparison between group A (difficult visualisation of RW) and B (easy visualisation of the RW) of the intersection point on the RWM of the prediction line. The intersection point was on the posteromedial part of the RW in most patients with a good intraoperative visualisation of the RW. Both groups consisted of 20 patients. $R W$ round window

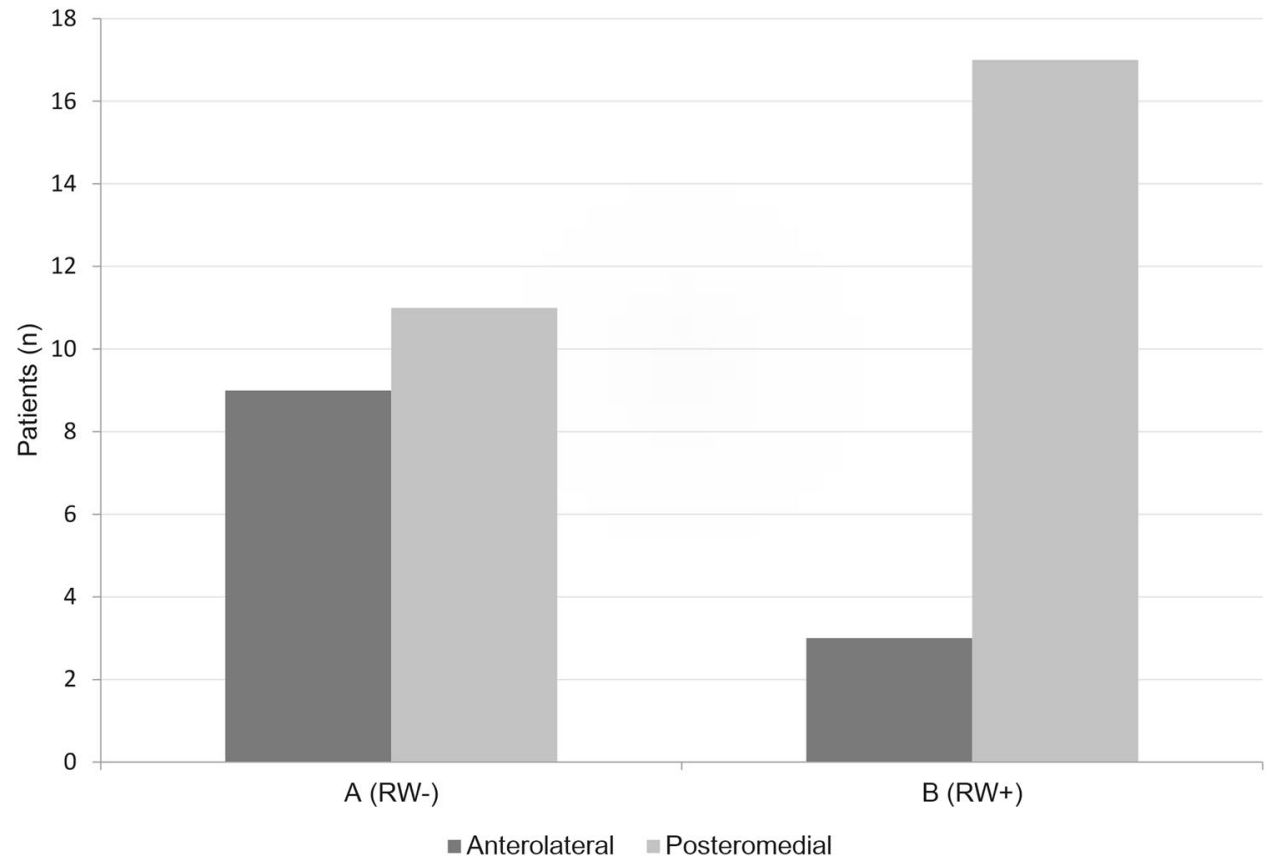




\section{Discussion}

\section{Operative report}

Our study shows that a direct RW approach is feasible in almost all cases (99\%). In addition, the RWM was difficult to visualize in $18 \%$ of the cases, usually because of a small facial recess $(n=26 / 28)$. In 13 cases ( $8 \%$ of total), the CTN had to be sacrificed to visualize the RWM. Clearly, in those cases, the CTN was limiting the viewing angle through the facial recess. In the remaining cases with a narrow facial recess the surgeon presumably succeeded in retaining the CTN, while implanting via the RWM. The retrospective design of this study, however, meant that we were limited to the retrospective operative reports, introducing possible bias. In addition, only crude estimations of the relevant outcomes were possible, i.e., we were only able to discern between easy and difficult cases.

Another study showed that direct RWM insertion is almost always possible, however, without reporting intraoperative events to important landmarks [14]. In contrast, other studies indicate that a direct RWM insertion is not always possible $[3,5,6,12,15]$. In these studies, the rate of unsuccessful direct RWM insertion ranges between 7 and $15 \%$, often necessitating a conventional cochleostomy.

The surgical approach in our study involved maximal exposure of the facial recess, while preserving the integrity of the FN (fallopian canal), CTN, posterior canal wall and bony tympanic annulus whenever possible, followed by drilling of the bony overhang of the RW niche to expose the RWM. The posterior canal wall was often thinned as much as possible. Subsequently, if needed, the CTN was sacrificed to visualize the RWM, potentially explaining the higher success rate of this study.

Other causes that can obscure the RWM visibility have been described in previous studies, such as a 'high riding' jugular bulb or an overhanging posterior canal wall $[6,12$, $16,17]$. In this study, there was one case with an overhanging posterior wall, and one with a high riding jugular bulb. In our cohort and in previous studies, the obscuration of the facial recess opening by the posterior wall or sigmoid sinus and jugular bulb is a rare phenomenon $(<1 \%)[14$, 18]. Some surgeons advocate in cases of an overhanging posterior wall, to "green stick fracture" the posterior wall medially (just lateral from the $\mathrm{FN}$ and push it forward) providing improved exposition of the RWM, access to the middle ear and perform implantation of the electrode array; then replace the (partly mobile) canal wall to its previous position where bone will regrow.

Lastly, we identified no cases with postoperative complaints related to the $\mathrm{CTN}$ or $\mathrm{FN}$, although patients with CTN lesions only mention their taste disturbances postoperatively if they are asked for it [19]. Other studies also showed that FN paralysis occurs infrequently $(<1 \%)$ following cochlear implantation procedure with a mastoidectomy-facial recess approach [7, 20]. In contrast, postoperative complications related to the CTN seem to occur more often $(>2 \%)$, although rates vary widely between studies [7, 19].

\section{Radiological measurements}

\section{Facial-chorda tympani nerve distance}

Comparison of the radiological measurements of the FNCTN distance between cases with normal and difficult visibility of RWM showed a smaller FN-CTN distance (difference of $0.7 \mathrm{~mm}$ ) for the cases with difficult visibility. Therefore, the FN-CTN measurements corresponded to the subjective outcome of the operative reports (i.e. small facial recess). These results show that the FN-CTN distance indeed provides a realistic estimate of the size of the 'window' to the middle ear structures [16]. A previous study also showed that the FN-CTN distance in the mastoid is important for the viewing angle through the facial recess opening [18]. Two other studies in adults showed no effect of the facial recess width on the visibility of the RW [10, 12]. These studies, however, measured the width of the facial recess using the posterior canal wall and FN. A correct facial recess opening, in our opinion, is the distance between the FN and CTN. By opting for the posterior wall, the mentioned study could have measured a facial recess width that was larger than what was actually possible intraoperatively.

\section{Prediction line}

Our study shows that the prediction line between the basal turn of the cochlea and the FN can be important in indicating the visibility of the RWM intraoperatively. A different study showed that the RWM visibility, classified into three types (invisible/nearly invisible, partially visible, fully visible), was predicted by a line drawn parallel to the external auditory canal and the FN [10]. The basal turn of the cochlea was in our experience more reliably and easier determined than a line parallel to the canal.

Previous studies have shown that the course of the FN can be highly heterogeneous, and might play a role in RWM visibility. In addition, the angle of rotation of the RWM plays an important role as well. These two aspects both heavily influence the outcome (anterolateral vs posteromedial intersection point) of our prediction line, confirming indeed their importance in determining the viewing angle of the RWM. 


\section{Clinical perspectives}

In this study, a RWM insertion approach was chosen for all patients if the ST and RWM were patent on the preoperative CT scan. The CTN was sacrificed if the RWM was difficult to recognize, achieving a high rate of direct RWM insertions. Other studies chose in such cases to convert the RWM approach to a conventional cochleostomy $[6,10]$. It is unclear which of these two options is the best choice for patients when the RWM is not or barely visible. On the one hand, opting for a conversion of insertion access to the cochlea by a conventional cochleostomy has its own potential downsides. An important rationale for direct RWM insertion, is that the RWM forms a natural gateway to the ST of the cochlea thereby preserving as much as possible the cochlear anatomy and inner ear microstructures. A cochleostomy also might lead to increased chance of translocation of the electrode array, or missing the ST altogether, leading to a direct scala vestibuli insertion, potentially negatively impacting the overall hearing outcomes of the CI user [21, 22]. Some surgeons, however, advocate that the vector of insertion angle might be more parallel and in line with the ST direction in the basal turn in contrast with RWM insertion. On the other hand, sacrificing the CTN can lead to symptoms such as a dry mouth and taste disorders [19, 23]. However, these symptoms might not always lead to persistent and troublesome complaints, and the recovery rate can be as high as 79\% after CTN lesion [19]. Probably the rate of postoperative complaints related to the CTN is underestimated, because most patients with CTN lesions only mention their taste disturbances postoperatively if they are asked for it.The high recovery rate of the CTN can be potentially explained by improved functioning of the ipsilateral glossopharyngeal nerve, re-innervation via contralateral or ipsilateral glossopharyngeal nerve and $\mathrm{CTN}$, and by subjective adaption of patients [19, 23]. Of course, both these options' advantages and disadvantages should be weighed against the specific clinical characteristics of the patient, e.g., in a patient with preoperative taste disturbances sacrificing the CTN would be contraindicated.

\section{Conclusion}

The RW approach for cochlear implantation seems feasible for most patients in our population. Difficult visualization of the RWM seems to lead to at least one intraoperative event in patients. The intraoperative events were iatrogenic damage of the CTN, (minor) exposure of the FN epineurium and lesions of the posterior canal wall. These patients had on the preoperative HRCT scan a smaller facial recess, and a more anterior position of the FN relative to the RW niche. These factors can be used to plan an insertion approach in cochlear implantation procedures, potentially leading to less iatrogenic damage of especially the CTN.

Funding This study was funded by Advanced bionics Corporation.

Availability of data and material Data sharing, including full protocol, participant datasets and statistical codes will be considered upon reasonable request.

\section{Compliance with ethical standards}

Conflict of interest The authors have no conflicts of interest to declare that are relevant to the content of this article.

Ethics approval Ethical approval was waived by the local Ethics Committee of UMC Utrecht in view of the retrospective nature of the study and all the procedures being performed were part of the routine care.

Consent to participate No specific informed consent was required.

Open Access This article is licensed under a Creative Commons Attribution 4.0 International License, which permits use, sharing, adaptation, distribution and reproduction in any medium or format, as long as you give appropriate credit to the original author(s) and the source, provide a link to the Creative Commons licence, and indicate if changes were made. The images or other third party material in this article are included in the article's Creative Commons licence, unless indicated otherwise in a credit line to the material. If material is not included in the article's Creative Commons licence and your intended use is not permitted by statutory regulation or exceeds the permitted use, you will need to obtain permission directly from the copyright holder. To view a copy of this licence, visit http://creativecommons.org/licenses/by/4.0/.

\section{References}

1. Mangus B, Rivas A, Tsai BS, Haynes DS, Roland JT Jr (2012) Surgical techniques in cochlear implants. Otolaryngol Clin N Am 45(1):69-80. https://doi.org/10.1016/j.otc.2011.08.017

2. Adunka OF, Pillsbury HC, Buchman CA (2010) Minimizing intracochlear trauma during cochlear implantation. Adv Otorhinolaryngol 67:96-107. https://doi.org/10.1159/000262601

3. Gazibegovic D, Bero EM (2017) Multicenter surgical experience evaluation on the Mid-Scala electrode and insertion tools. Eur Arch Otorhinolaryngol 274(2):1147-1151. https://doi. org/10.1007/s00405-016-4255-3

4. Richard C, Fayad JN, Doherty J, Linthicum FH Jr (2012) Round window versus cochleostomy technique in cochlear implantation: histologic findings. Otol Neurotol 33(7):1181-1187. https://doi. org/10.1097/MAO.0b013e318263d56d

5. Gudis DA, Montes M, Bigelow DC, Ruckenstein MJ (2012) The round window: is it the "cochleostomy" of choice? Experience in 130 consecutive cochlear implants. Otol Neurotol 33(9):14971501. https://doi.org/10.1097/MAO.0b013e31826a52c7

6. Leong AC, Jiang D, Agger A, Fitzgerald-O'Connor A (2013) Evaluation of round window accessibility to cochlear implant insertion. Eur Arch Otorhinolaryngol 270(4):1237-1242. https:// doi.org/10.1007/s00405-012-2106-4

7. Hansen S, Anthonsen K, Stangerup SE, Jensen JH, Thomsen J, Caye-Thomasen P (2010) Unexpected findings and surgical 
complications in 505 consecutive cochlear implantations: a proposal for reporting consensus. Acta Otolaryngol 130(5):540-549. https://doi.org/10.3109/00016480903358261

8. Vaid S, Vaid N (2014) Imaging for cochlear implantation: structuring a clinically relevant report. Clin Radiol 69(7):e9-e24. https ://doi.org/10.1016/j.crad.2014.03.011

9. Harnsberger HR, Dart DJ, Parkin JL, Smoker WR, Osborn AG (1987) Cochlear implant candidates: assessment with CT and MR imaging. Radiology 164(1):53-57. https://doi.org/10.1148/radio logy.164.1.3108956

10. Chen J, Wu Y, Shi J, Jia H, Wang Z, Zhang Z, Wu H (2019) Predictors of round window membrane visibility in pediatric cochlear implant surgery using temporal bone HRCT: a retrospective study. Int J Pediatr Otorhinolaryngol 121:150-153. https://doi. org/10.1016/j.ijporl.2019.03.017

11. Karkas A, Champfleur NM, Uziel A, Mondain M, Puel JL, Venail F (2018) Benefit of preoperative temporal bone CT for atraumatic cochlear implantation. Otol Neurotol 39(3):e186-e194. https:// doi.org/10.1097/MAO.0000000000001692

12. Kashio A, Sakamoto T, Karino S, Kakigi A, Iwasaki S, Yamasoba $\mathrm{T}$ (2015) Predicting round window niche visibility via the facial recess using high-resolution computed tomography. Otol Neurotol 36(1):e18-23. https://doi.org/10.1097/MAO.0000000000000644

13. Park E, Amoodi H, Kuthubutheen J, Chen JM, Nedzelski JM, Lin VY (2015) Predictors of round window accessibility for adult cochlear implantation based on pre-operative CT scan: a prospective observational study. J Otolaryngol Head Neck Surg 44:20. https://doi.org/10.1186/s40463-015-0073-7

14. Bae SC, Shin YR, Chun YM (2019) Cochlear implant surgery through round window approach is always possible. Ann Otol Rhinol Laryngol 128(6_suppl):38S-44S. https://doi. org/10.1177/0003489419834311

15. Jang JH, Choo OS, Kim H, Yi Park H, Choung YH (2019) Round window membrane visibility related to success of hearing preservation in cochlear implantation. Acta Otolaryngol 139(7):618624. https://doi.org/10.1080/00016489.2019.1609701

16. Hamamoto M, Murakami G, Kataura A (2000) Topographical relationships among the facial nerve, chorda tympani nerve and round window with special reference to the approach route for cochlear implant surgery. Clin Anat 13(4):251-256. https://doi.org/10.1002/1098-2353(2000)13:4\%3c251 ::AID-CA4\%3e3.0.CO;2-E

17. Xie LH, Tang J, Miao WJ, Tang XL, Li H, Tang AZ (2018) Preoperative evaluation of cochlear implantation through the round window membrane in the facial recess using high-resolution computed tomography. Surg Radiol Anat 40(6):705-711. https://doi. org/10.1007/s00276-018-1972-x

18. Lee DH, Kim JK, Seo JH, Lee BJ (2012) Anatomic limitations of posterior tympanotomy: what is the major radiologic determinant for the view field through posterior tympanotomy? J Craniofac Surg 23(3):817-820. https://doi.org/10.1097/SCS.0b013e3182 $4 \mathrm{e} 6 \mathrm{ca} 7$

19. Ziylan F, Smeeing DPJ, Bezdjian A, Stegeman I, Thomeer H (2018) Feasibility of preservation of chorda tympani nerve during noninflammatory ear surgery: a systematic review. Laryngoscope 128(8):1904-1913. https://doi.org/10.1002/lary.26970

20. Jeppesen J, Faber CE (2013) Surgical complications following cochlear implantation in adults based on a proposed reporting consensus. Acta Otolaryngol 133(10):1012-1021. https://doi. org/10.3109/00016489.2013.797604

21. O'Connell BP, Cakir A, Hunter JB, Francis DO, Noble JH, Labadie RF, Zuniga G, Dawant BM, Rivas A, Wanna GB (2016) Electrode location and angular insertion depth are predictors of audiologic outcomes in cochlear implantation. Otol Neurotol 37(8):1016-1023. https://doi.org/10.1097/MAO.0000000000 001125

22. Wanna GB, Noble JH, Carlson ML, Gifford RH, Dietrich MS, Haynes DS, Dawant BM, Labadie RF (2014) Impact of electrode design and surgical approach on scalar location and cochlear implant outcomes. Laryngoscope 124(Suppl 6):S1-7. https://doi. org/10.1002/lary.24728

23. McManus LJ, Stringer MD, Dawes PJ (2012) Iatrogenic injury of the chorda tympani: a systematic review. J Laryngol Otol 126(1):8-14. https://doi.org/10.1017/S0022215111002039

Publisher's Note Springer Nature remains neutral with regard to jurisdictional claims in published maps and institutional affiliations. 